\title{
VOLUMEN DE HUMEDECIMIENTO POR LA APLICACIÓN DE HIDROGEL EN SUELOS DE DIFERENTES TEXTURAS
}

\author{
EFFECTS OF HYDROGEL ON MOISTURE VOLUME IN SOILS WITH DIFFERENT \\ TEXTURES
}
Rubén Darío Rivera Fernández ${ }^{1 *}$, Carlos Mora Mueckay²®, Juan Ramón Moreira Salto ${ }^{1}{ }^{\oplus}$ y Dídimo Alexander Mendoza Intriago ${ }^{3}{ }^{\oplus}$

\author{
${ }^{1}$ Carrera de Agropecuaria. Universidad Laica Eloy Alfaro de Manabí - Extensión Chone, Av. Marcos Aray Dueñas, 130301, \\ Chone, Ecuador. \\ ${ }^{2}$ Universidad Técnica de Manabi, Av. Urbina, y Portoviejo, 130105, Portoviejo, Ecuador. \\ ${ }^{3}$ Carrera de Agronomía. Universidad Laica Eloy Alfaro de Manabi, Av. Circunvalación 130804, Manta, Ecuador. \\ *Autor para correspondencia: ruben.rivera@uleam.edu.ec
}

Manuscrito recibido el 23 de septiembre del 2019. Aceptado, tras revisión, el 14 de septiembre de 2020. Publicado el 1 de marzo de 2021.

\begin{abstract}
Resumen
El estudio tuvo como objetivo determinar el volumen de humedecimiento (bulbo húmedo) del hidrogel aplicado en tres tipos de suelo. Se utilizaron suelos: a) arcilloso (arcilla 52\%; limo 32\%; arena 16\%); b) franco arcilloso limoso (arcilla 36\%; limo 56\%; arena 6\%) c) franco arenoso (arcilla 12\%; limo 32\%; arena 56\%), a los cuales se aplicó hidrogel al 1\% de potasio. La aplicación se realizó con hidrogel previamente hidratado, en tres diámetros que fueron: 4.7, 7.0 y $10.5 \mathrm{~cm}$ con una longitud de $10 \mathrm{~cm}$ donde se obtuvieron los siguientes volúmenes iniciales 173.5, 384.8 y $866 \mathrm{~cm}^{3}$ que ocupaba el hidrogel. Se midió el volumen de humedecimiento $\left(\mathrm{cm}^{3}\right)$, porcentaje de humedad y la hidratación del hidrogel en el suelo. Los resultados indican que el volumen de humedecimiento depende del volumen inicial, de manera que a mayor volumen inicial se tendrá mayor volumen de humedecimiento indiferente del tipo de suelo; sin embargo, el suelo franco arenoso presenta un mayor volumen de humedecimiento, seguramente por la movilidad del agua en el mismo. La zona humedecida incrementa su humedad en un $14 \%$ indiferentemente del tipo de suelo. La hidratación del hidrogel en el suelo solo alcanza un $42 \%$ en comparación con la hidratación en agua libre. La determinación del volumen de humedecimiento permite estimar la cantidad y ubicación de hidrogel que se debe aplicar en un cultivo en función del bulbo que se requiere formar en el suelo.
\end{abstract}

Palabras clave: Hidratación en suelo, bulbo húmedo, incremento de humedad, franco arenoso. 


\begin{abstract}
The objective of the study was to determine the wetting volume (wet bulb) of the hydrogel applied to three types of soil. Soils used were: a) clay (clay $52 \%$; silt $32 \%$; sand $16 \%$ ); b) silty clay loam (36\% clay; $56 \%$ silt; $6 \%$ sand) c) sandy loam ( $12 \%$ clay; $32 \%$ silt; $56 \%$ sand), to which $1 \%$ potassium hydrogel was applied. The application was made with previously hydrated hydrogel, in three diameters that were: $4.7,7.0$ and $10.5 \mathrm{~cm}$ with a length of $10 \mathrm{~cm}$ where the following initial volumes were obtained 173.5, 384.8 and $866 \mathrm{~cm}^{3}$ occupied by the hydrogel. The wetting volume $\left(\mathrm{cm}^{3}\right)$, moisture percent, and the hydration of the hydrogel in the soil were measured. The results indicate that the humidification volume depends on the initial volume, so the higher the initial volume, the greater the humidification volume regardless of the type of soil; however, it presents a greater volume of humidification in the sandy loam soil, probably due to mobility of the water in it. The moistened area increases its humidity by $14 \%$ regardless of the type of soil. The hydration of the hydrogel in the soil only reaches $42 \%$ compared to hydrating it in free water. Determining the volume of wetting allows estimating the amount and location of hydrogel to be applied to a crop based on the bulb that needs to be formed in the soil.
\end{abstract}

Keywords: Soil hydration, wet bulb, increased humidity, sandy loam.

Forma sugerida de citar: $\quad$ Rivera-Fernández, R.D., Mora-Mueckay, C., Moreira-Salto, J.R. y Mendoza-Intriago, D.A. (2021). Volumen de humedecimiento por la aplicación de hidrogel en suelos de diferentes texturas. La Granja: Revista de Ciencias de la Vida. Vol. 33(1):67-75. http:/ / doi.org/10.17163/lgr.n33.2021.06.

\title{
IDs Orcid:
}

Rubén Darío Rivera Fernández: http:/ / orcid.org/0000-0003-2436-1321

Carlos Mora Mueckay: http:/ /orcid.org/0000-0001-8714-4653

Juan Ramón Moreira Salto: http:/ / orcid.org/0000-0003-4434-5986

Dídimo Alexander Mendoza Intriago: http:/ / orcid.org/0000-0002-6524-3228 


\section{Introducción}

El hidrogel es usado en la agricultura tanto por sus beneficios económicos como agronómicos (Cisneros y col., 2020), en hortalizas (Pereira y col., 2019) y en cultivos perennes (M'barki y col., 2019). El hidrogel es un polímero hidrófilo, blando, elástico con capacidad de expandirse con el agua, aumentando su peso, pero sin perder su estructura, que en estado deshidratado son cristales (Ahmed, 2015). Sus bondades le han permitido ser usado en áreas como la biomedicina (Nicolson y Vogt, 2001; Amin, Rajabnezhad y Kohli, 2009) y en la agricultura se ha utilizado como elemento para evitar el estrés hídrico en los cultivos (Dragusin y col., 1996) ya que retener agua es sin duda su principal característica (Satriani, Catalano y Scalcione, 2018). Los primeros estudios en esta área datan en los años 70s en la germinación y crecimiento de semillas (Palacios y col., 2016).

Por el momento se conoce la cantidad de aplicación, las mimas que son variadas en función del cultivo y sustrato o suelo a utilizar. Agaba y col. (2011) recomiendan 0,4\% de hidrogel en Agrostis stolonifera y $2 \%$ en suelos arenosos. Jankowski, Jankowska y Sosnowski (2011) aplicaron $50 \mathrm{gm}^{2}$ en diferentes sustratos y cultivos. Rivera y col. (2018) encontraron que con la aplicación de entre 2 y 2.5 $\mathrm{g} /$ planta en el cultivo de pimiento alcanzaron los mayores rendimientos. Aunque los estudios donde se muestra una alta influencia del hidrogel en los cultivos corresponden cuando hay altas cantidades de hidrogel. Maldonado-Benitez y col. (2011) recomiendan $4 \mathrm{gL}^{-1}$ o mayor, y Chirino, Vilagrosa y Vallejo (2011) mencionan una dosis de 1,5\% de hidrogel para obtener una alta efectividad.

Por otro lado, el comportamiento del hidrogel está ligado a las características físicas del suelo en especial la textura (Rivera y Mesías, 2018), lo cual muestra la dinámica del comportamiento del mismo. El hidrogel ha sido muy utilizado en suelos de baja retención de agua (Lopes y col., 2013; Rojas y col., 2004; Idrobo, Rodríguez y Díaz, 2010), también en áreas agrícolas de escasez de precipitaciones o sequias (Santelices, 2005), en donde existen varios tipos de textura del suelo. Además, los experimentos realizados en su mayoría han sido en macetas y en mezclas de sustratos o suelo disturbado (Maldonado-Benitez y col., 2011; Idrobo, Rodríguez y Díaz, 2010; Najafi, Golchin y Mohebi, 2013; Jankowski, Jankowska y Sosnowski, 2011) lo cual puede tener un comportamiento diferente al aplicarlo en el campo directamente. Fonteno y Bilderback (1993) afirman que la efectividad del hidrogel está determinada por el tipo de suelo o sustrato, básicamente por su porosidad. Barón y col. (2007) mencionan que el hidrogel modifica la dinámica hidráulica del suelo. (Orikiriza y col., 2013) identificaron que el hidrogel provocó una mayor supervivencia de $\mathrm{Pi}$ cea abies, Pinus sylivestris y Fagus sylvatica en suelos arenosos.

De igual forma, frecuentemente se relaciona el hidrogel con el manejo del recurso hídrico en los cultivos, en lo referente al intervalo de riego (Wadas y col., 2010; Yazdani, Allahdadi y Akbari, 2007) en especial en el establecimiento de cultivos forestales (Hüttermann, Zommorodi y Reise, 1999; AlHumaid y Moftah, 2007; Agaba y col., 2010). Sin embargo, también hay estudios que demuestran que la presencia del hidrogel no influye significativamente. Wang (1989) al estudiar la aplicación de hidrogel en Codiaeum variegatum encontró que no existe un crecimiento diferente comparado con el control; sin embargo, si evita su marchitamiento. Geesing y Schmidhalter (2004), indican que la aplicación de hidrogel no muestra ningún beneficio en la supervivencia o el crecimiento de Triticum aestivum. Esta variación de criterios hace creer que existen aspectos no considerados al momento del uso de hidrogel en los cultivos. Entre ellos esta área o volumen de humedecimiento (bulbo húmedo) que provoca el hidrogel en el suelo, lo cual sería necesario para saber la ubicación del producto en el suelo. Las raíces de las plantas pueden ampliar su sistema radicular con mayor volumen de humedad en el suelo, lo que permitiría hacerlo más eficiente, incluso cuando se coloca el producto de forma localizada en los cultivos. Con este antecedente, el objetivo fue determinar el volumen de humedecimiento del hidrogel en diferentes diámetros y tipos de suelos.

\section{Materiales y métodos}

\subsection{Material experimental}

El estudio se realizó en el Laboratorio de suelo y agua de la Universidad Laica Eloy Alfaro de Manabí, Chone-Ecuador. Se utilizó como material experimental al hidrogel tipo agrícola NewGel G, distri- 
buido por Marketing del Ecuador y fabricado por Whidden Industrial Park, el mismo que presenta la siguiente composición: Polímero de Acrilamina de potasio al $99,9 \%$ y potasio $0,01 \%$. Se utilizó suelo de origen fluvial (Mollic Udifluvent.) y coluvial (Vertic Hapluodoll). Se realizó un análisis de textura por el método de pipeteo para separar las partículas de arena limo y arcilla y la clasificación textural por el triángulo de textura (Moorberg y Crouse, 2017). Se utilizaron para el estudio tres clases texturales: a) arcilloso (arcilla 52\%; limo 32\%; arena $16 \%$ ); b) franco arcilloso limoso (arcilla 36\%; limo 56\%; arena 6\%) c) franco arenoso (arcilla 12\%; limo 32\%; arena 56\%).

Tabla 1. Promedios de los parámetros finales del orifico donde se colocó el hidrogel en los diferentes suelos.

\begin{tabular}{lccc}
\hline \multicolumn{4}{c}{ Suelo Franco Arenoso } \\
\hline Diámetro final $(\mathrm{cm})^{*}$ & $9,3 \pm 0,9$ & $12,8 \pm 1,1$ & $17,5 \pm 1,6$ \\
Longitud final $(\mathrm{cm})^{*}$ & $13,2 \pm 0,2$ & $13,7 \pm 0,1$ & $1,7 \pm 0,3$ \\
Volumen humedecido $\left(\mathrm{cm}^{3}\right)^{*}$ & $723,2 \pm 24,2$ & $902 \pm 35,2$ & $1539,4 \pm 56,3$ \\
\hline \multicolumn{4}{c}{ Suelo franco arcilloso limoso } \\
\hline Diámetro final $(\mathrm{cm})^{*}$ & $9,02 \pm 0,8$ & $13 \pm 0,7$ & $14,22 \pm 1,1$ \\
Longitud final $(\mathrm{cm})^{*}$ & $13 \pm 1,8$ & $12,3 \pm 1,3$ & $12,2 \pm 1,2$ \\
Volumen humedecido $\left(\mathrm{cm}^{3}\right)^{*}$ & $657,2 \pm 22,4$ & $1247,8 \pm 54,2$ & $1071,6 \pm 48,6$ \\
\hline \multicolumn{4}{c}{ Suelo Arcilloso } \\
\hline Diámetro final $(\mathrm{cm})^{*}$ & $7,1 \pm 0,6$ & $10,6 \pm 1,1$ & $14,06 \pm 1,7$ \\
Longitud final $(\mathrm{cm})^{*}$ & $12,4 \pm 1,8$ & $12,3 \pm 2,0$ & $12,5 \pm 1,9$ \\
Volumen humedecido $\left(\mathrm{cm}^{3}\right) *$ & $317,4 \pm 23,6$ & $700,6 \pm 31,1$ & $1074,8 \pm 49,3$ \\
\hline \multirow{4}{*}{$*$ Parámetros iniciales: Diámetro $(\mathrm{cm}): 4,7 ; 7 ; 10,5 ;$ Longitud: $10 \mathrm{~cm}}$. \\
Volumen: 173,5; 384,8; $865,9 \mathrm{~cm}^{3} ;$ valores respectivamente de cada columna.
\end{tabular}

\subsection{Manejo experimental}

Para la ejecución del experimento se utilizó un recipiente trasparente (vidrio) que permitió visualizar la humedad que provocaba el hidrogel en el suelo. Este mismo tuvo una capacidad de $0,027 \mathrm{~m}^{3}$ con dimensiones de 0,30 m en todos sus lados. En el cual se colocaron las muestras de los diferentes tipos de suelo. Las muestras de suelo fueron tomadas y secadas previa la colocación en los recipientes.

\subsection{Volumen de humedecimiento}

Para medir esta variable se utilizó hidrogel previamente hidratado según lo recomendado por Rivera y Mesías (2018), quienes sugieren $100 \mathrm{~mL}$ de agua por cada gramo de hidrogel. Posteriormente se colocó en orificios que poseían los siguientes diámetros 4,$7 ; 7,0$ y $10,5 \mathrm{~cm}$ y a una profundidad de 15 $\mathrm{cm}$, de los cuales $10 \mathrm{~cm}$ fueron ocupados dejando 5 $\mathrm{cm}$ de suelo que cubrían al producto. Posteriormente se dejó por 24 horas al ambiente sin protección de los rayos solares. Se medió la distancia del humedecimiento desde los bordes del producto hasta los cambios de tonalidad oscura provocada por la humedad del hidrogel en el suelo, obteniendo el diámetro $(\mathrm{cm})$, longitud $(\mathrm{cm})$ y el volumen $\left(\mathrm{cm}^{3}\right)$; se calculó ajustando los valores a la ecuación de un cilindro (1), obteniendo un volumen inicial de 173,5; 384,8 y $866 \mathrm{~cm}^{3}$.

$$
V=\pi * r^{2} * h
$$

Donde, $\mathrm{V}$ es el volumen en $\mathrm{cm}^{3}, \mathrm{r}$ es la radio del orifico $(\mathrm{cm})$ y h es la longitud $(\mathrm{cm})$ del orificio contenido con hidrogel.

\subsection{Humedad del suelo}

El porcentaje de humedad se midió antes y después (24 horas) de aplicado el hidrogel. Se tomó la muestra de la sección del suelo humedecida por el hidrogel y por gravimetría se determinó el porcentaje de humedad. Se determinó el incremento de la humedad, tomando en cuenta la humedad inicial del suelo y la humedad final. Este procedimiento se llevó a cabo en cada tipo de suelo y por triplicado. 


\subsection{Hidratación del hidrogel en el suelo}

Para estimar la hidratación del hidrogel en el suelo, se colocó $2 \mathrm{~g}$ de hidrogel sin hidratar en un tamiz \#60, al cual se ubicó en suelo seco de los diferentes tipos suelo en estudio. Con este procedimiento se evitó que se disperse el hidrogel al momento de hidratarse y medir su hidratación. Posteriormente se aplicó agua suficiente simulando el riego por superficie y goteo, hasta lograr el suelo a capacidad de campo. Después de una hora de aplicado el agua de riego se retiró el tamiz con hidrogel y se separó el hidrogel hidratado del suelo, se pesó y estimó el porcentaje de hidratación, en comparación con la hidratación en agua libre.

\section{Resultados y Discusión}

\subsection{Volumen de humedecimiento del suelo}

En la Tala 1 se puede apreciar los parámetros finales de la sección humedecida del suelo en los diferentes suelos. Se observa que el diámetro final de hume- decimiento está en función del diámetro inicial, con cierta tendencia a aumentar mientras mayor sea el diámetro inicial. La longitud varió debido a un aumento del humedecimiento de la parte inferior del bulbo inicial; en la parte superior no presentó un aumento considerable, el mismo que fue depreciable. El aumento en la parte inferior tuvo menor variación con respecto al diámetro, aumentando hasta 4,7 cm en el suelo franco arenoso. Estos parámetros evidencian el movimiento del agua vertical y horizontal en el suelo, el mismo que está directamente relacionado con la característica textural del suelo, lo cual fue evidente al evaluar esta variable en los diferentes suelos. Por otro lado, el volumen final tuvo un aumento considerable y el mismo estuvo en función del tipo de suelo, siendo el suelo franco arenoso el de mayor humedecimiento, coincidiendo con lo mencionado por Rivera y Mesías (2018), quienes relacionan el comportamiento del hidrogel con la textura del suelo y esta a su vez con la superficie especificada del suelo (Ruiz y col., 2016) que también depende de la textura del suelo. Narjary y col. (2012), mencionan que el hidrogel suele ser más eficiente en suelo arenosos que en suelos pesados.

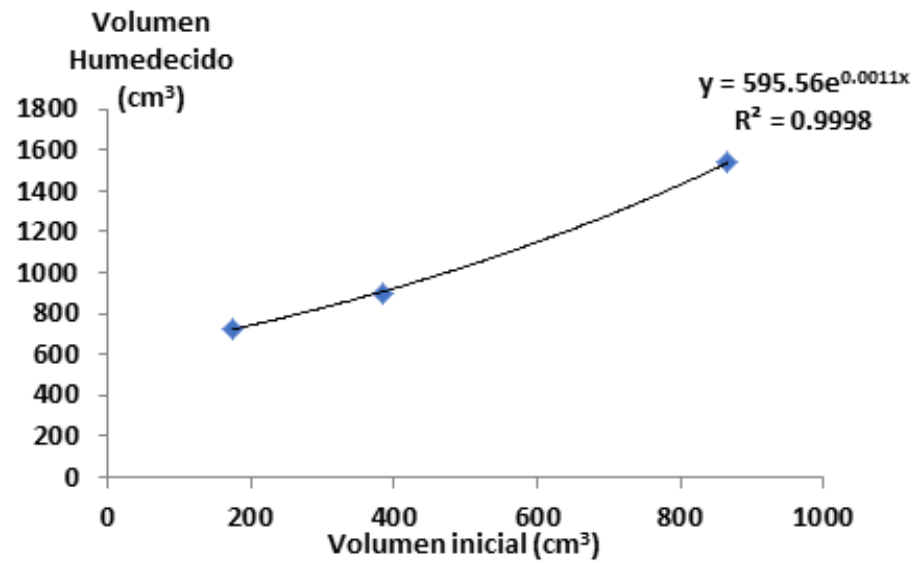

Figura 1. Relación entre volumen inicial y el volumen humedecimiento final en el suelo arenoso.

Los suelos arenosos tienen una mayor facilidad para la infiltración del agua en comparación con los suelos pesados, de manera que se puede deducir que el agua contenida en la cápsula de hidrogel, cuando pasa al suelo, tiene un comportamiento similar al aplicar agua de riego, con la diferencia que las cantidades de agua contenidas en el hidrogel son pequeñas comparadas al riego tradicional y que es- ta entrega de agua al suelo ocurre cuando el suelo ejerce una alta presión matricial y cuando el suelo tiene menor humedad, lo cual es consistente debido a que el menor contenido de humedad inicial (franco-arenoso) lo presentó el suelo de mayor volumen de humedecimiento. No obstante, esta conjetura debe ser corroborada en experimentos diseñados para este fin, dado que no fue considerado en es- 
ta investigación. Por otro lado, el volumen inicial es directamente proporcional al volumen de humedecimiento, aunque esta condición solo se cumplió en el suelo franco arenoso y arcilloso (Figura 1 y 3), en cambio el suelo franco arcilloso limoso no presentó una tendencia (Figura 2). Es necesario tener en claro que la determinación del bulbo húmedo formado por el hidrogel es importante para decidir dónde colocar el producto en función del sistema radicular, con el fin de estimar la cantidad de hidrogel a aplicar y el diámetro y profundidad del orificio donde se pretenda colocar el hidrogel para que las raíces puedan captar agua para su desarrollo.

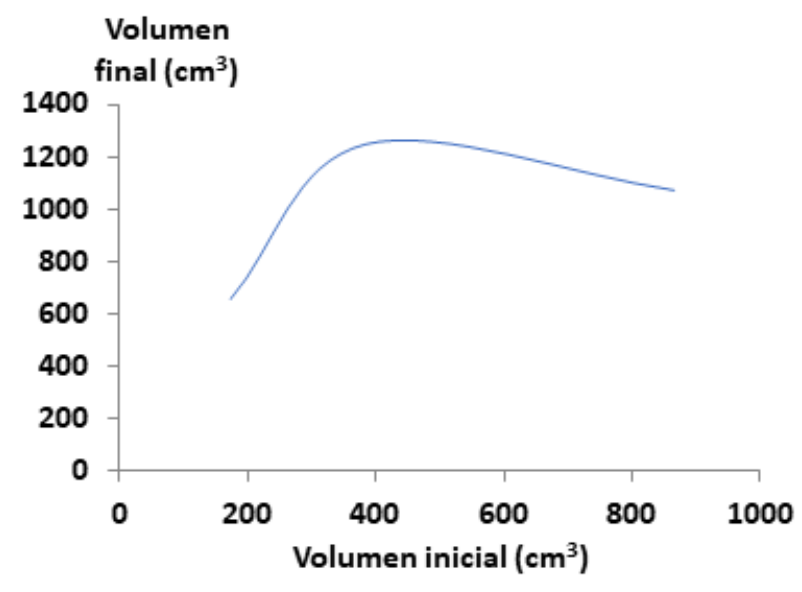

Figura 2. Tendencia del volumen inicial y final en el suelo franco.

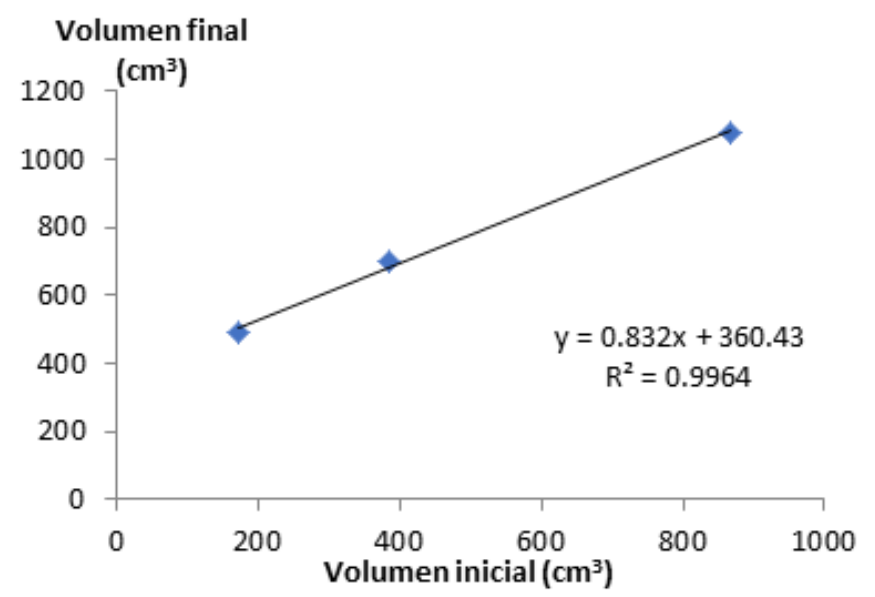

Figura 3. Tendencia de la relación entre el volumen inicial y final en el suelo arcilloso.

\subsection{Humedad del suelo}

El porcentaje de humedad de la zona de humedecimiento del suelo se presenta en el Tabla 2, donde se observa que el incremento de la humedad es de alrededor de $14 \%$ sin importar el tipo de suelo. Estos valores son similares a los encontrados por Rivera y Mesías (2018) aunque difieren en el suelo franco donde el incremento que encontraron fue de $17,4 \%$. Con esta tendencia se podría sugerir un incremento del $14 \%$ de la humedad del suelo en un suelo seco. Es posible que el suelo, al tener baja humedad, refleje de mejor manera la humedad del suelo por la acción del hidrogel, por tanto, en necesario realizar estudio con diferentes niveles de humedad del suelo. 
Tabla 2. Aumento de la humedad con la aplicación de hidrogel en los tres suelos.

\begin{tabular}{ccc}
\hline Tipos de suelo & $\begin{array}{c}\text { Humedad inicial \% } \\
\text { (Suelo seco) }\end{array}$ & $\begin{array}{c}\text { Porcentaje de } \\
\text { humedecimiento (\%) }\end{array}$ \\
\hline Arenoso & 4,7 & $18,8 \pm 0,8$ \\
Franco & 6,5 & $20,8 \pm 1,2$ \\
Arcilloso & 8,6 & $22,8 \pm 1,5$ \\
\hline
\end{tabular}

\subsection{Hidratación del hidrogel en el suelo}

La hidratación del hidrogel del suelo es menor al del porcentaje de la hidratación en agua (Figura 4), apenas llega al $42 \%$ con respecto a la hidratación en agua. De manera que si se pretende colocar el hidrogel en el suelo sin previa hidratación se debe tener en cuenta su capacidad de hidratar al suelo y por ende a la planta.

Este porcentaje de hidratación responde inde- pendientemente del tipo de riego que se realice. La información referente a esta hidratación del hidrogel en el suelo no está definida en la literatura científica, aunque se recomienda ampliamente en los cultivos, principalmente en los forestales. El hecho que no se hidrate en igual proporción que la hidratación que sucede en agua libre indica que es posible que el suelo realice una resistencia, logrando que el hidrogel no se expanda normalmente y por ende no absorba el agua a plenitud.

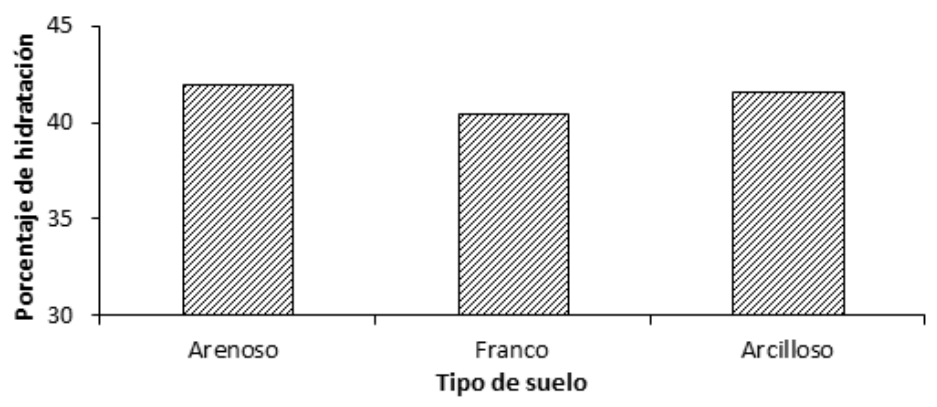

Figura 4. Porcentaje de hidratación del hidrogel en el suelo con respecto a la hidratación en agua libre.

\section{Conclusiones}

El volumen de humedecimiento del hidrogel ocurre en función del tipo de suelo y del volumen inicial del hidrogel en el suelo. Con un comportamiento exponencial, el suelo franco arenoso presenta mayor facilidad para formar un mayor bulbo húmedo a diferencia de los demás suelos. La zona humedecida por el hidrogel tiene un incremento de la humedad alrededor del $14 \%$ con respecto al contenido inicial, indiferente del tipo de suelo, con la posibilidad de llevar al suelo a capacidad de campo.

Al aplicar hidrogel sin hidratar en el suelo, éste no podrá hidratarse con la misma magnitud que en agua libre, debido a que el suelo ejerce presión y dificulta que la partícula de hidrogel se hidrate normalmente. En todos los suelos se logró una hidratación del $42 \%$ con respecto a la hidratación en agua libre. Es importante considerar que el conocer el comportamiento del hidrogel en el suelo optimizará su uso, por tanto, se deben considerar estudios sobre su estabilidad, rehidratación y duración en el suelo con cultivos en producción.

\section{Referencias}

Agaba, H. y col. (2011). «Hydrogel amendment to sandy soil reduces irrigation frequency and improves the biomass of Agrostis stolonifera». En: 
Agricultural Sciences 2.04, 544. Online:https: / / bit.ly/3m6MKZg.

Agaba H.and Baguma Orikiriza, L. y col. (2010). «Effects of hydrogel amendment to different soils on plant available water and survival of trees under drought conditions». En: Clean-Soil, Air, Water 38.4, 328-335. Online:https: / / bit.ly / 2J3KZ0U.

Ahmed, E. (2015). «Hydrogel: Preparation, characterization, and applications: A review». En: Journal of advanced research 6.2, 105-121. Online:https: / / bit.ly/368Sdct.

Al-Humaid, A. y A. Moftah (2007). «Effects of hydrophilic polymer on the survival of buttonwood seedlings grown under drought stress». En: Journal of Plant Nutrition 30.1, 53-66. Online:https:/ / bit.ly/3q0lLB7.

Amin, S., S. Rajabnezhad y K. Kohli (2009). «Hydrogels as potential drug delivery systems». En: Scientific Research and Essays 4.11, 1175-1183. Online:https://bit.ly/37cxaFk.

Barón, A. y col. (2007). «Evaluación de hidrogeles para aplicaciones agroforestales». En: Ingeniería e Investigación 27.3, 35-44. Online:https:/ / bit.ly/ 2JfcnbQ.

Chirino, E., A. Vilagrosa y V. R. Vallejo (2011). «Using hydrogel and clay to improve the water status of seedlings for dryland restoration». En: Plant and Soil 344.1-2, 99-110. Online:https: //bit.ly/3mfboHk.

Cisneros, E. y col. (2020). «Efecto de los polímeros en la economía del agua». En: Revista Ingeniería Agrícola 10.1, 21-27. Online:http: / / opn. to / a / $\mathrm{uApBC}$.

Dragusin, M. y col. (1996). «Hydrogels used for medicine and agriculture». En: Gels. Springer, 123-125. Online:https:/ / bit.ly/3fFlVc8.

Fonteno, W. y T. Bilderback (1993). «Impact of hydrogel on physical properties of coarsestructured horticultural substrates». En: Journal of the American Society for Horticultural Science 118.2, 217-222. Online:https:/ / bit.ly/3fFmR08.

Geesing, D. y U. Schmidhalter (2004). «Influence of sodium polyacrylate on the water-holding capacity of three different soils and effects on growth of wheat». En: Soil use and management 20.2, 207-209. Online:https:/ / bit.ly/39kL4YB.

Hüttermann, A., M. Zommorodi y K. Reise (1999). "Addition of hydrogels to soil for prolonging the survival of Pinus halepensis seedlings subjected to drought». En: Soil and tillage research 50.3-4, 295-304, Online:https:/ / bit.ly /2V81tdh.

Idrobo, H., A. Rodríguez y J. Díaz (2010). «Comportamiento del hidrogel en suelos arenosos». En: Ingeniería de Recursos Naturales y del Ambiente 9, 33-37. Online:https://bit.ly/33kSVS0.

Jankowski, K., J. Jankowska y J. Sosnowski (2011). «Rooting properties of lawn grasses established on the basis of red fescue in the aspect of the applied hydrogel». En: Acta Scientiarum Polonorum. Agricultura 10.4, 69-78. Online:https: / / bit.ly / 36dDFs1.

Lopes, J. y col. (2013). «Sobrevivência e crescimento inicial de pinhão-manso em função da época de plantio e do uso de hidrogel». En: Ciência Florestal 23.3, 489-498. Online:https: / / bit.ly / 3nZYUDS.

Maldonado-Benitez, K. y col. (2011). «Producción de Pinus greggii Engelm. en mezclas de sustrato con hidrogel y riego, en vivero». En: Agrociencia 45.3, 389-398. Online:https:/ / bit.ly/3mii2g2.

Moorberg, Colby J. y David A. Crouse (2017). Soils Laboratory Manual, K-State Edition. URL: https:/ / newprairiepress.org/ebooks/15.

M'barki, N. y col. (2019). «Cultivar dependent impact of soil amendment with water retaining polymer on olive (Olea europaea L.) under two water regimes». En: Agricultural Water Management 216, 70-75. Online:https : / / bit . ly / $2 \mathrm{HMv} 06 \mathrm{E}$

Najafi, F., A. Golchin y M. Mohebi (2013). «The effects of Aquasorb water-absorbing polymer and irrigation frequency on yield, water use efficiency and growth indices of greenhouse cucumber». En: J. Sci. E Technol. Greenhouse Culture 4.15, 14-22. Online:https://bit.ly/3q8LqHB.

Narjary, B. y col. (2012). "Water availability in different soils in relation to hydrogel application». En: Geoderma 187, 94-101. Online:https://bit.ly/ 2V87TX0.

Nicolson, P. y J. Vogt (2001). «Soft contact lens polymers: an evolution». En: Biomaterials 22.24, 3273-3283. Online:https:/ / bit.ly/36aEMsD.

Orikiriza, L. y col. (2013). «Effects of hydrogels on tree seedling performance in temperate soils before and after water stress». En: Journal of Environmental Protection 4.07, 713-721. Online:https: / / bit.ly/3o2FNZT.

Palacios, A. y col. (2016). «Hidrogel como mitigador de estrés hídrico: una revisión.» En: Revista Ibe- 
roamericana de Ciencias 3.5, 80-90. Online:https: //bit.ly/39nnk5Z.

Pereira, B. y col. (2019). «Watermelon initial growwth under diferent hydrogel concentrations and shading conditions.» En: Revista Caatinga 32.4, 915-923. Online:https : / / bit . ly / $3 \mathrm{mdKvnb.}$

Rivera, R. y F. Mesías (2018). «Absorción de agua de hidrogel de uso agrícola y su humedecimiento de tres tipos de suelo». En: Revista de la Facultad de Ciencias Agrarias 50.2, 15-21. Online:https: //bit.ly/3919aCw.

Rivera, R. y col. (2018). «Hydrogel for improving water use efficiency of Capsicum annun crops in Fluvisol soil». En: Revista de la Facultad de Ciencias Agrarias 50.2, 23-31. Online:https: / / bit.ly / 3919aCw.

Rojas, B. y col. (2004). «Estudio de la germinación de semillas de tomate en suelos áridos extraidos de la peninsula de Araya (Venezuela) al utilizar polímeros de tipo hidrogeles». En: Revista Iberoamericana de Polímeros 5.1, 17-27. Online:https:/ / bit.ly/3o1IYkr.

Ruiz, H. A. y col. (2016). «La superficie específica de oxisoles y su relación con la retención hídrica». En: Revista de la Facultad de Ciencias Agrarias.
Universidad Nacional de Cuyo. Mendoza. Argentina. 48.2, 95-105. Online:https: / / bit.ly/39s9EHf.

Santelices, R. (2005). «Desarrollo de una plantación de Eucalyptus globulus establecida en primavera con diferentes tratamientos de riego». En: Bosque (Valdivia) 26.3, 105-112. Online:https: / / bit. ly/3mg982n.

Satriani, A., M. Catalano y E. Scalcione (2018). «The role of superabsorbent hydrogel in bean crop cultivation under deficit irrigation conditions: A case-study in Southern Italy». En: Agricultural Water Management 195, 114-119. Online:https: / / bit.ly/3o2h4EV.

Wadas, J. y col. (2010). «Uso de hidrogel na sobrevivência de mudas de Eucalyptus urograndis produzidas com diferentes substratos e manejos hídricos.» En: Ciência Florestal 20.2, 217-224. Online:https://bit.ly/3q90OE8.

Wang, Y. (1989). "Medium and hydrogel affect production and wilting of tropical ornamental plants». En: HortScience 24.6, 941-944. Online:.

Yazdani, F., I. Allahdadi y G. A. Akbari (2007). «Impact of superabsorbent polymer on yield and growth analysis of soybean (Glycine max L.) under drought stress condition.» En: Pakistan journal of biological sciences: PJBS 10.23, 4190-4196. Online:https:/ / bit.ly/3fHKCol. 